\title{
Clinical significance of metabolism-related genes and FAK activity in ovarian high-grade serous carcinoma
}

\author{
Masakazu Sato", Sho Sato, Daisuke Shintani, Mieko Hanaoka, Aiko Ogasawara, Maiko Miwa, Akira Yabuno,
} Akira Kurosaki, Hiroyuki Yoshida, Keiichi Fujiwara and Kosei Hasegawa

\begin{abstract}
Background: Administration of poly (ADP-ribose) polymerase (PARP) inhibitors after achieving a response to platinum-containing drugs significantly prolonged relapse-free survival compared to placebo administration. PARP inhibitors have been used in clinical practice. However, patients with platinum-resistant relapsed ovarian cancer still have a poor prognosis and there is an unmet need. The purpose of this study was to examine the clinical significance of metabolic genes and focal adhesion kinase (FAK) activity in advanced ovarian high-grade serous carcinoma (HGSC).

Methods: The RNA sequencing (RNA-seq) data and clinical data of HGSC patients were obtained from the Genomic Data Commons (GDC) Data Portal and analysed (https://portal.gdc.cancer.gov/). In addition, tumour tissue was sampled by laparotomy or screening laparoscopy prior to treatment initiation from patients diagnosed with stage IIIC ovarian cancer (International Federation of Gynecology and Obstetrics (FIGO) classification, 2014) at the Saitama Medical University International Medical Center, and among the patients diagnosed with HGSC, 16 cases of available cryopreserved specimens were included in this study. The present study was reviewed and approved by the Institutional Review Board of Saitama Medical University International Medical Center (Saitama, Japan). Among the 6307 variable genes detected in both The Cancer Genome Atlas-Ovarian (TCGA-OV) data and clinical specimen data, 35 genes related to metabolism and FAK activity were applied. RNA-seq data were analysed using the Subio Platform (Subio Inc, Japan). JMP 15 (SAS, USA) was used for statistical analysis and various types of machine learning. The Kaplan-Meier method was used for survival analysis, and the Wilcoxon test was used to analyse significant differences. $P<0.05$ was considered significant.
\end{abstract}

Results: In the TCGA-OV data, patients with stage IIIC with a residual tumour diameter of 1-10 $\mathrm{mm}$ were selected for $\mathrm{K}$ means clustering and classified into groups with significant prognostic correlations $(p=0.0444)$. These groups were significantly associated with platinum sensitivity/resistance in clinical cases $\left(x^{2}\right.$ test, $\left.p=0.0408\right)$ and showed significant relationships with progression-free survival $(p=0.0307)$.

Conclusion: In the TCGA-OV data, 2 groups classified by clustering focusing on metabolism-related genes and FAK activity were shown to be associated with platinum resistance and a poor prognosis.

Keywords: Ovarian cancer, Platinum resistance, Machine learning

\section{${ }^{*}$ Correspondence: masakasatou-scc@saitama-pho.jp}

Department of Gynecologic Oncology, Saitama Medical University

International Medical Center, 1397-1 Yamane, Hidaka, Saitama 350-1298, Japan

\section{Background}

Gynecological malignancies include cervical cancer, uterine cancer, and ovarian cancer, among others. Ovarian cancer is the 5th leading cause of cancer deaths among 
women worldwide and is considered to have an extremely poor prognosis [1-3]. One of the reasons for the poor prognosis is that most patients are asymptomatic, and most cases are discovered at an advanced stage, i.e., with dissemination or metastasis in the abdominal cavity [4]. Although the prognosis of ovarian cancer patients has dramatically improved since the advent of paclitaxel and carboplatin combination therapy (TC therapy), the prognosis is still poor for advanced stage III and IV patients, who account for $60 \%$ of ovarian cancer patients [4-7]. One of the reasons for the poor prognosis of patients with advanced stage is the tendency for relapse. Ovarian cancer is reported to respond well to initial treatment (platinum drugs including carboplatin as mentioned above); however, approximately half of cases will relapse [1]. Since achieving a radical cure is difficult after relapse, treatment after relapse mainly aims to prolong survival and alleviate symptoms [5-9]. Thus, treatments that do not cause relapse or metastasis and treatments that provide hope for remission even after relapse/metastasis are urgently needed.

Recently, clinical trials have shown that administration of poly (ADP-ribose) polymerase (PARP) inhibitors to ovarian cancer patients after achieving a response to platinum-containing drugs significantly prolonged relapsefree survival compared to placebo administration. PARP inhibitors are used in actual clinical practice [10-17]. Thus, a promising medication has emerged for platinumsensitive patients. However, the prognosis of platinumresistant patients is still poor. Thus, new drugs must be developed because platinum sensitivity or platinum resistance cannot be identified without administration of a platinum-containing drug. If a method is developed to predict platinum resistance or platinum sensitivity before administration, proper treatment can be offered to each individual patient $[18,19]$.

The involvement of cancer stem cells (CSCs) in cancer relapse and treatment resistance has been reported in recent years, indicating that cancer tissues are heterogeneous and that some cancer cells, such as CSCs, are involved in relapse and treatment resistance [20-23]. Even if non-cancer stem cells (non-CSCs) are treated, they can lead to relapse as long as a CSC is alive. Conversely, if CSCs are eradicated, the remained cancer tissue (non-CSCs) will eventually be eliminated by host antitumor immunity. From the results of RNA sequencing (RNA-seq) and metabolomic analysis using cell lines, the authors found that the metabolic pathway and Focal adhesion kinase (FAK) activity associated with CSCs for gynecologic cancer may differ from those of non-CSCs [24].

Therefore, the purpose of this study was to examine the clinical significance of metabolic genes and FAK activity in advanced ovarian high-grade serous carcinoma (HGSC). Specifically, RNA-seq was performed on cancer specimens before treatment initiation to examine relationships with the effects of platinumcontaining drugs with an emphasis on metabolic genes and FAK activity. Machine learning including cluster analysis was used for analysis.

Using machine learning, predicting prognoses for cancer patients and the therapeutic effects of platinum-containing drugs can be widely performed [25-36]. In this study, by showing that the therapeutic effect can be predicted using metabolic genes and FAK activity, these variables were confirmed to be clinically significant.

\section{Methods}

\section{Patient and sample collection}

The present study was reviewed and approved by the Institutional Review Board of Saitama Medical University International Medical Center (approval no.13165). Patients diagnosed with ovarian cancer stage IIIC (International Federation of Gynecology and Obstetrics (FIGO) classification 2014) who started treatment at Saitama Medical University International Medical Center between November 2008 and August 2016 were targeted. There were 101 patients with HGSC who had stage IIIC tumours in that period, and tumour tissue sampling was performed during open surgery or exploratory laparoscopy before treatment initiation. Among them, representative 16 cases with available cryopreserved specimens were analysed.

Tumour specimens were collected by surgery and immediately cryopreserved at $-80{ }^{\circ} \mathrm{C}$. Total RNA was extracted as previously reported [37]. In brief, RNA was extracted from the frozen tissues using NucleoSpin RNA (Takara, Japan). Quality control was performed using a Bioanalyzer (Agilent, USA), and all RNA integrity number (RIN) values were $>8.0$.

The clinical information of the 16 cases were obtained from the electrical health record, and is shown in Table 1. Platinum-based neoadjuvant chemotherapy (NAC) was performed as primary treatment, and an interval debulking surgery (IDS) was performed when the effect was confirmed. The Response Evaluation Criteria in Solid Tumours (RECIST) were used to determine the therapeutic effect [38]. As a guideline to measure the effect of chemotherapy, the period from administration of the last platinum-containing chemotherapy until disease deterioration (platinum-free interval, PFI) was examined [39]. 
Table 1 Clinical specimen data

\begin{tabular}{llllll}
\hline Sample & Age & $\begin{array}{l}\text { Treatment } \\
\text { effect }\end{array}$ & Vital status & PFI (Months) & Clusters \\
\hline 1 & 66 & PD & 1 & 4 & 2 \\
2 & 54 & CR & 1 & 4 & 2 \\
3 & 59 & PD & 1 & 6 & 1 \\
4 & 66 & CR & 1 & 0 & 2 \\
5 & 75 & SD & 1 & 7 & 2 \\
6 & 56 & PR & 1 & 5 & 2 \\
7 & 70 & CR & 1 & 0 & 1 \\
8 & 62 & PR & 0 & 0 & 2 \\
9 & 72 & PR & 1 & 42 & 1 \\
10 & 44 & PR & 0 & 22 & 1 \\
11 & 70 & PR & 1 & 21 & 2 \\
12 & 71 & CR & 0 & 34 & 1 \\
13 & 66 & PR & 0 & 29 & 1 \\
14 & 52 & PR & 0 & 17 & 1 \\
15 & 66 & CR & 0 & 37 & 1 \\
16 & 54 & CR & 0 & 24 & 2 \\
\hline
\end{tabular}

Sixteen patients diagnosed with ovarian high-grade serous carcinoma (HGSC), stage IIIC (FIGO classification 2014), who had started treatment were analysed $C R$ complete response, $P R$ partial response, $S D$ stable disease, $P D$ progressive disease

Vital status: alive $=0$, dead $=1$. PFI Platinum-free interval. Cluster: Cluster classified by cluster analysis

\section{Data analysis}

RNA-seq data were analysed using the Subio Platform (Subio Inc, Japan) [52].

\section{TCGA-OV data}

The read count value data were analysed. Normalization/ preprocessing was performed as follows. For log transformation, the read count value was converted to a logarithm with a base of 2 . If the read count was 0 , a missing value was documented. Subsequently, global normalization was performed with the 90th percentile. Then, for the low signal cutoff, if the value after normalization was less than 50, it was replaced with 50 and used as the cutoff value. To account for missing values, original read counts of 0 , indicating a missing value, were assigned a value of 2 to the 5 th power.

For centring, the expression level of each gene was converted to the ratio against the average value. The value generated by applying the above normalization and preprocessing is displayed as a value called the Processed Signal on the Subio Platform and is the log2 ratio against the average value of the expression levels of all samples for each gene.

Measurement values with a read count less than 100 were considered to be unreliable, and genes with a read count value less than 50 were excluded from the analysis in 189 samples, reflecting half of the 378 samples. Thus, 16,485 genes were extracted.

\section{Clinical specimen data}

Similar to the TCGA-OV data, the clinical specimen data were normalized and preprocessed. However, the processing method is fine-tuned on the basis of sample size and the distribution of read count values.

For log transformation, the read count value was converted to a logarithm with a base of 2 . However, if the read count value was 0 , logarithmic transformation was not possible, and the result was replaced with a missing value. Subsequently, global normalization was performed through alignment with the 75 th percentile. Then, when the value after normalization was smaller than 100 (low signal cutoff), it was replaced with 100 . To account for missing values, sites with a missing value due to an original read count of 0 were assigned a value of 2 to the 6th power.

The value generated by applying the above processing is displayed as the Processed Signal on the Subio Platform as well as the TCGA-OV data. Measurement values with a read count value less than 100 were considered to be unreliable, and these genes were removed. To exclude genes whose expression did not change and mary tumour samples and 5 recurrent tumour samples were included. Seq dataset consisted of 378 samples. A total of 373 pri-

\section{The cancer genome atlas-ovarian (TCGA-OV)} mons (GDC) Data Portal (https://portal.gdc.cancer. gov/) [41-51].

RNA seq data for ovarian cancer patients available at TCGA were extracted on October 30, 2019. The RNA- 
genes whose expression changed randomly, genes whose average Processed Signal was in the range of -0.3 to 0.3 were removed. Thus, 6840 genes were extracted.

Finally, the Processed Signal of 6307 genes, which was extracted from TCGA-OV data and clinical sample data, was selected as a candidate of the variable to be used in the machine learning analysis. There are many genes related to FAK pathways and metabolism, however, selecting many variables for machine learning could result in overfitting [53]. And we focused on major metabolic and FAK pathway genes related to such as glycolysis, Krebs cycle, serine metabolism, glutamine metabolism and integrins [54-58].

\section{Statistical analysis}

JMP 15 (SAS, USA) was used for statistical analysis and various types of machine learning. The Kaplan-Meier method was used for survival analysis, and the Wilcoxon test was used to analyse significant differences. $P<0.05$ was considered significant.

\section{Results}

\section{TCGA-OV data}

TCGA-OV data included data from ovarian cancer patients with advanced stage I to stage IV disease, but since the prognosis differs depending on the stage of advancement, in this study, we analysed the data for the patients with stage III ovarian cancer. However, in the treatment of ovarian cancers, the prognosis differs depending on the amount of residual tumour at the time of surgery [59]. In other words, in the treatment of ovarian cancer, surgery resulting in no residual tumour is considered complete surgery with a good prognosis, while surgery resulting in a residual tumour exceeding $1 \mathrm{~cm}$ in diameter is considered suboptimal surgery without a good prognosis. Surgery resulting in a residual tumour with a diameter within $1-10 \mathrm{~mm}$ is considered optimal surgery. In practice, even in the TCGA-OV data, as shown in Fig. 1, the prognosis was poor depending on the amount of residual tumour during surgery. In other words, when considering the relationship between the prognosis and biological characteristics of cancer tissue, the results may differ depending on the residual tumour diameter. In this study, the medical case with a residual tumour measuring between 1-10 $\mathrm{mm}$ was extracted and analysed. The clinical information including the prognosis of 130 cases was obtained and studied.

\section{Classification by cluster analysis}

In 130 cases obtained as described above, cluster analysis was performed for gene expression, as shown in Table 2. The selection of genes is described in the Introduction and Discussion. The genes related to metabolism and FAK activity were studied.

A total of 130 cases were classified into 2 groups (Fig. 2) according to $\mathrm{K}$ means clustering [60]. As shown in Fig. 3, the results were classified into 2 groups, which were significantly related to prognosis (Wilcoxon-test, $p=$

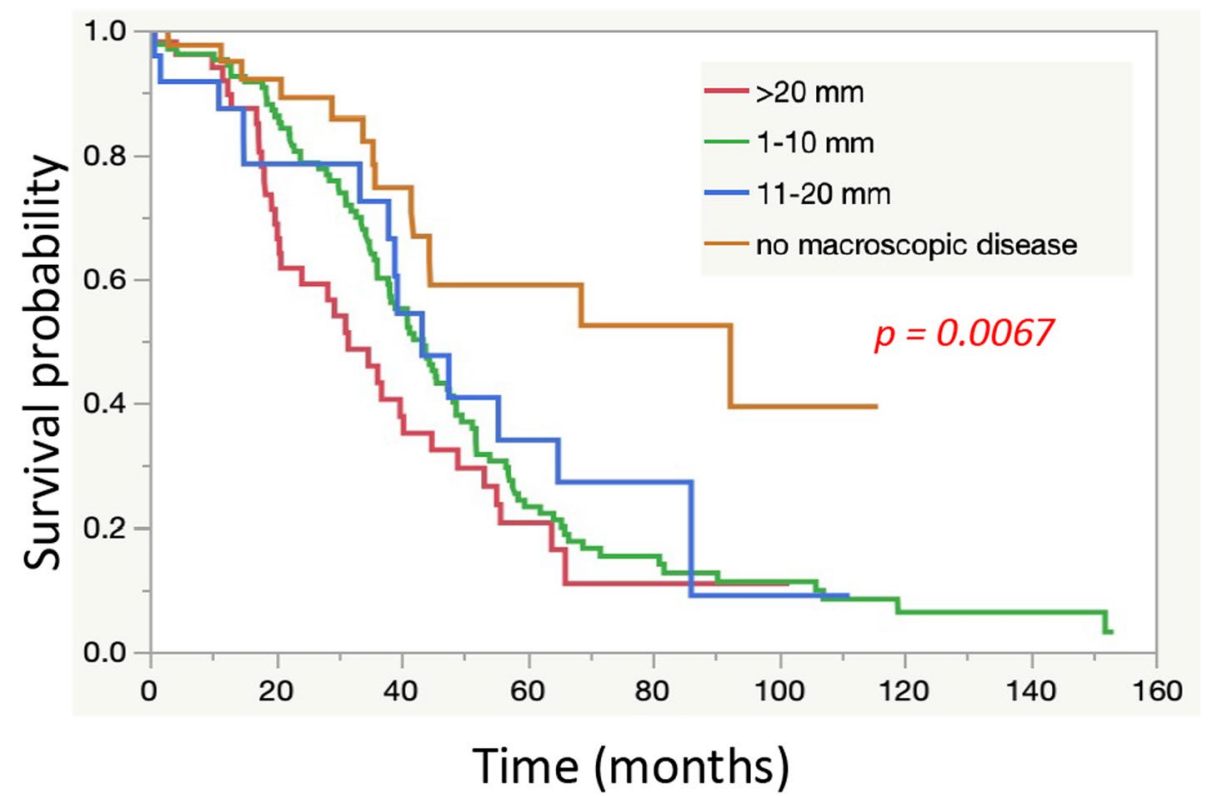

Fig. 1. Relationship between a Residual Tumour at the Time of Surgery and the Prognosis of Patients with Advanced Stage IIIC. A larger residual tumour diameter corresponds to a worse prognosis $(p=0.0067)$ 
Table 2 Genes used for clustering analysis

\begin{tabular}{lllll}
\hline Gene name & & & & \\
\hline CAV1 & GLUD1 & GOT1 & GPT & GPT2 \\
HK1 & HOOK1 & ITGA1 & ITGA11 & ITGA2 \\
ITGA3 & ITGA4 & ITGA5 & ITGA6 & ITGA7 \\
ITGA9 & ITGAL & ITGAM & ITGAV & ITGAX \\
ITGB1 & ITGB2 & ITGB3 & ITGB4 & ITGB5 \\
ITGB6 & LDHA & LDHB & PHGDH & PSAT1 \\
PSPH & ROCK2 & SLC1A5 & SLC7A5 & SRC
\end{tabular}

Among the 6307 genes detected in both TCGA-OV data and clinical specimen data for FAK activity, 35 genes related to metabolism were analysed

$0.0444)$. The mean value and manifestation of each gene in these groups are shown in Fig. S4. Regarding metabolic genes, both high and low expression levels and the overall balance were involved in the metabolic phenotype [61]. Therefore, in this examination, the difference between these 2 groups was unclear.

\section{Analysis including clinical specimen data}

Subsequently, similar clustering including clinical specimen data was performed with the TCGA-OV data. Only 4 cases among 130 cases were classified differently from the abovementioned clustering (Fig. 4). Actually, almost similar results were obtained regarding prognosis (Fig. 5). The relationship between platinum resistance/sensitivity in clinical specimens and this classification is shown in Table 1. In this examination, samples 1-8 are defined as platinum resistant, and samples 9-16 are defined as platinum sensitive. Generally, sample 5 is defined as platinum sensitive because the PFI is 7 months $>6$ months. However, the median PFI according to this examination was 12 months. Therefore, sample 5 was defined as platinum resistant in this study. In clusters 1 and 2, cluster 2 was significantly associated with platinum resistance (Fig. 6 and Table 1 , $\mathrm{X}^{2}$ test, $\left.p=0.0408\right)$.

In this classification, progression-free survival (PFS) after platinum-containing drug administration was examined, and a significant correlation was found (Fig. 7a, $p=$ 0.0307). In other words, the group classified as cluster 2 had a significantly shorter PFS than the group classified as cluster 1 in the clinical data. Further, cluster 2 had a worse prognosis tendency with respect to the overall survival (OS) in the clinical data. However, a significant difference was not observed (Fig. $7 \mathrm{~b}, p=0.0638$ ).

\section{Discussion}

By using machine learning including deep learning, in recent years, many studies on applying machine learning in cancer research have been performed [26, 29, $30,33,62,63]$. Using machine learning, predicting the prognoses of ovarian cancer patients and the therapeutic effects of platinum-containing drugs can be widely performed [64-72]. In most cases, machine learning from results such as RNA-seq results is first applied $[26,30]$. After extracting the gene cluster related to prognosis, the significance is examined using pathway analysis. These methods can accurately predict the

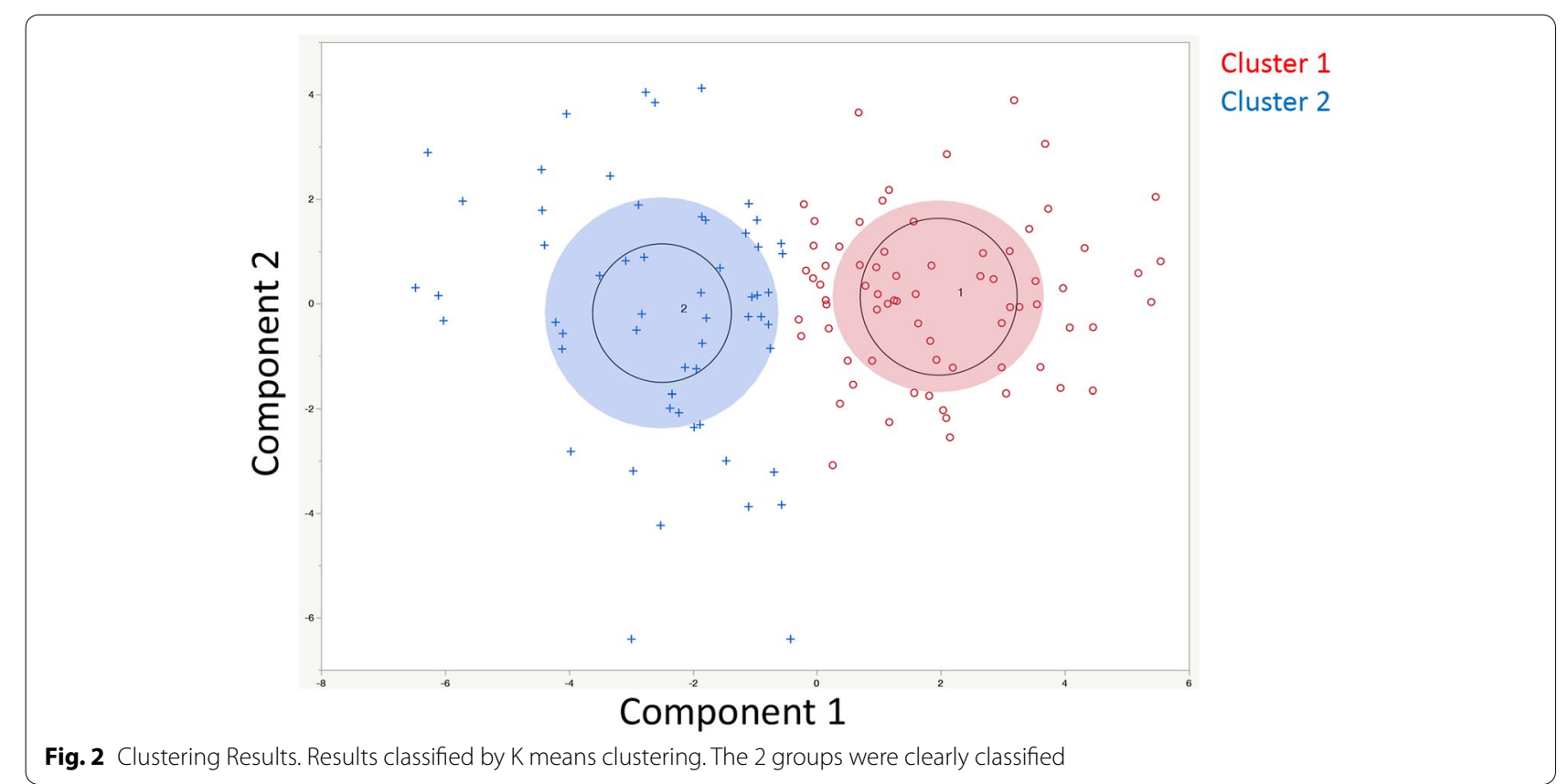




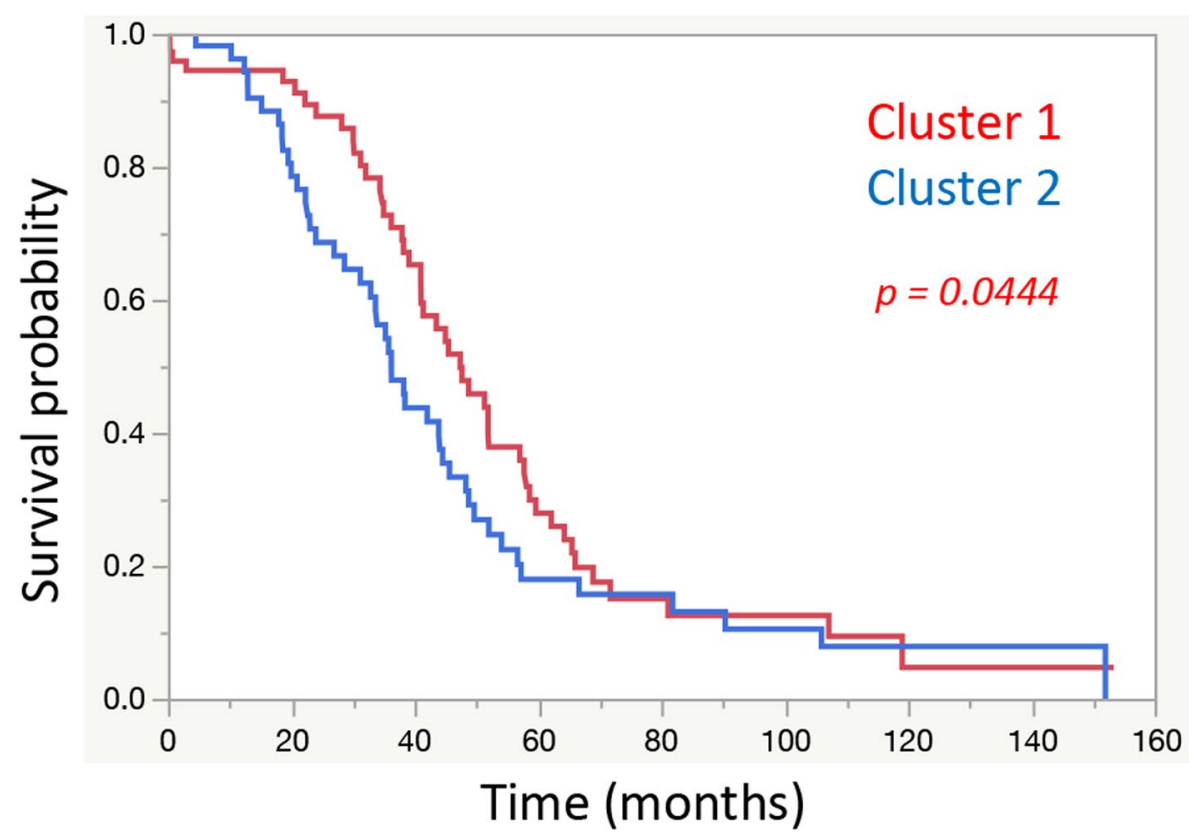

Fig. 3 Relationships with Prognosis based on Clustering Results. Among the clusters classified by K means clustering, cluster 2 had a significantly worse prognosis than cluster $1(p=0.0444)$

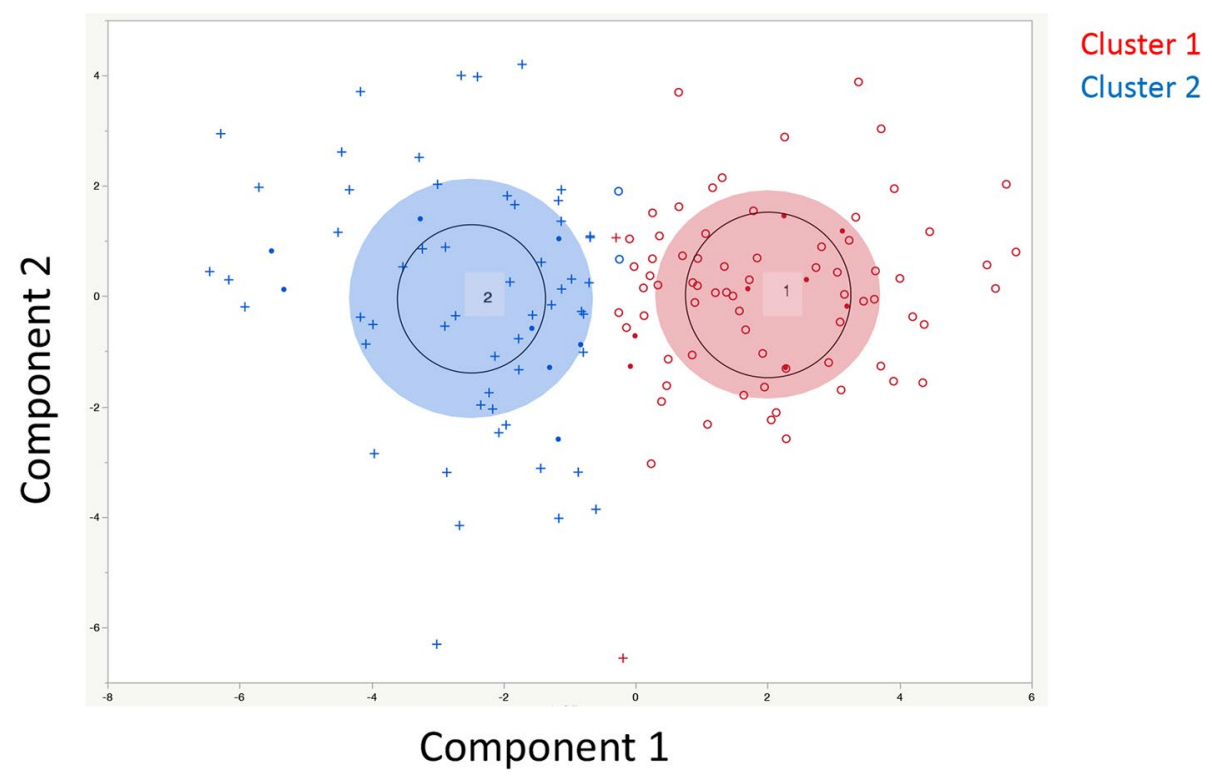

Fig. 4 Clustering Results including Clinical Specimen Data. The results were almost the same as those in Fig. 2. The red' + ' indicates items classified as cluster 2 in Fig. 2. The blue 'O' indicates items classified as cluster 1 in Fig. 2. Only 4 cases had a cluster classification different from the classification in Fig. 2. '•' indicates the results of clinical specimens

prognosis. In fact, when the analysis was performed similarly to this examination, after focusing on the group of genes in references, platinum resistance/sensitivity could be significantly predicted (Table $S 1, \chi^{2}$ test, $p=0.0023)$. The effects of platinum-containing drugs can be accurately predicted by homologous recombination deficiency (HRD) scores [67, 68]. In these predictions, many pathways are used for prediction, or 


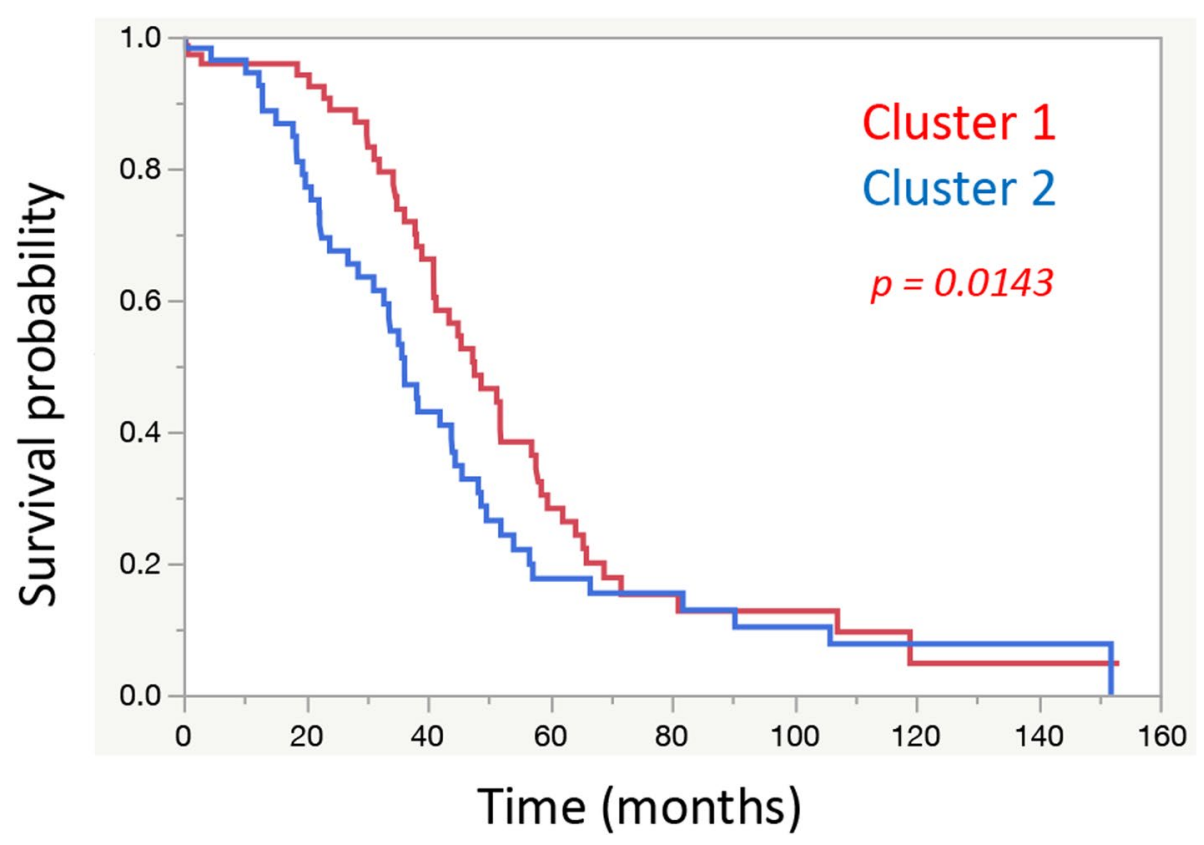

Fig. 5 Relationships with Prognosis based on Clustering Results including Clinical Specimen Data (TCGA OV Data). Similar to Fig. 2, among the clusters classified by K means clustering, cluster 2 had a significantly worse prognosis than cluster $1(p=0.0143)$

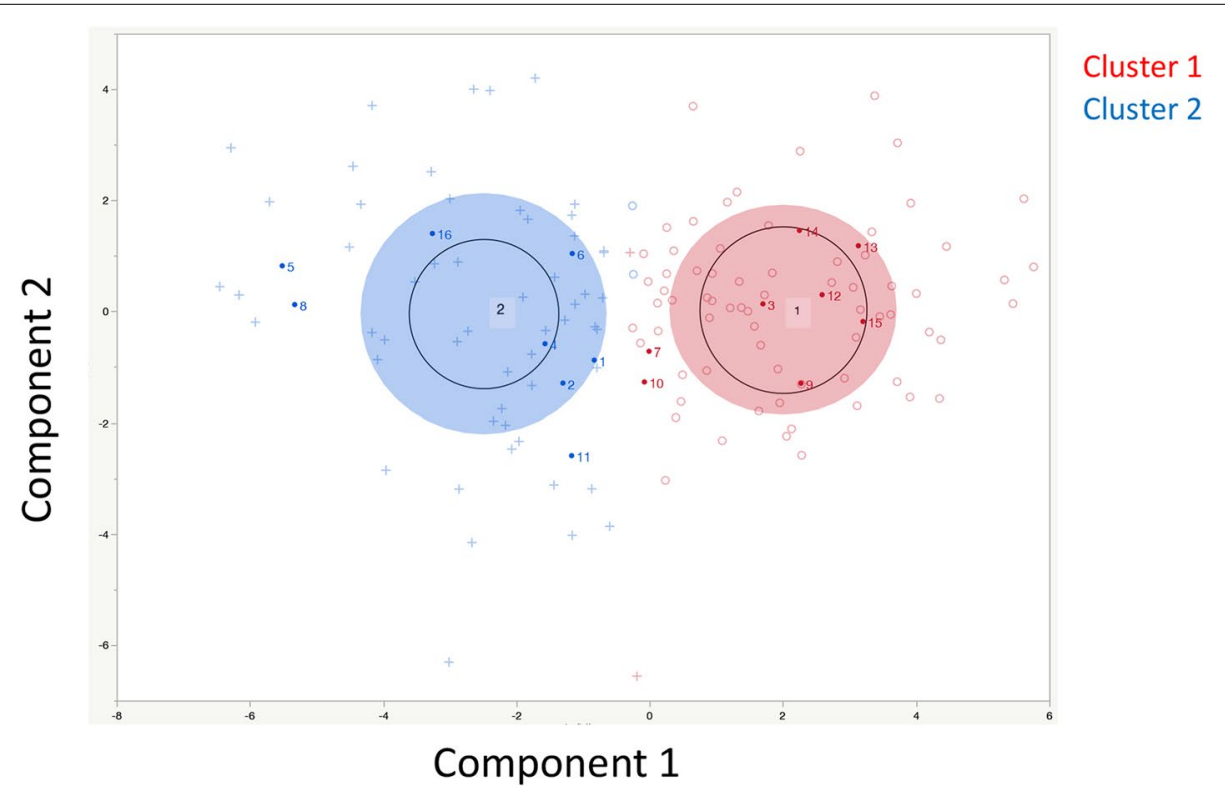

Fig. 6 Clustering Results for Clinical Specimens. Each number is the sample number in Table 1

several cases already awaiting treatment are used [31]. This examination focuses only on gene expression levels related to metabolic pathways and the FAK pathway identified in previous basic experiments and therefore differs from the other examinations. We applied machine learning including neural networks, however,
$\mathrm{K}$ means clustering was the best to classify groups of platinum resistance/sensitivity in our cases.

In recent years, metabolism in cancer has received considerable attention with the development and popularization of metabolomic analysis [73-76]. Metabolic changes reflect expression levels at the cellular level, and 

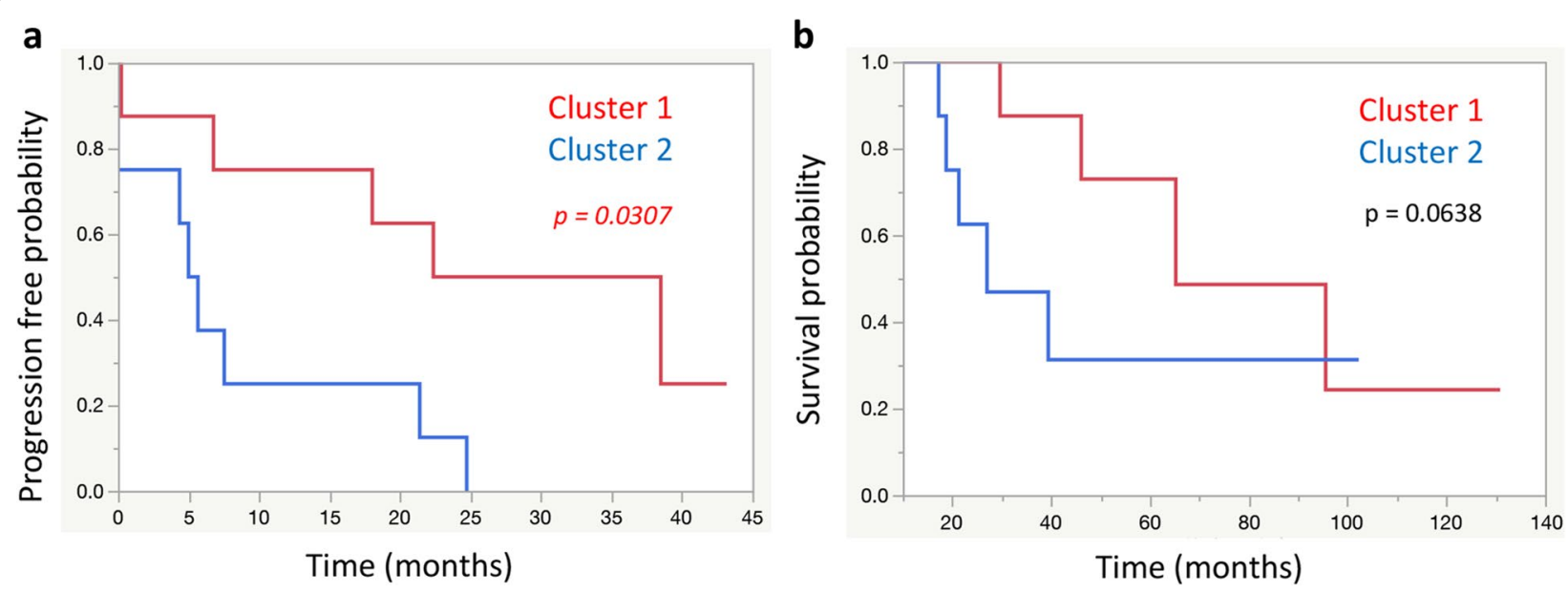

Fig. 7 Relationship between Clustering Results including Clinical Specimen Data and Clinical Data. a Progression free survival (PFS) based on Clustering Results including Clinical Specimen Data. Cluster 2 had a significantly worse prognosis than cluster 1 in terms of PFS ( $p=0.0307)$. $\mathbf{b}$ Overall survival (OS) based on Clustering Results including Clinical Specimen Data. No significant difference in OS were found between cluster 1 and cluster $2(p=0.0638)$

this analysis is closely related to how a cell behaves in the body (that is, whether a cell is highly malignant). In fact, references and self-study cases indicate that targeting the metabolic pathway may have a therapeutic effect on chemotherapy-resistant ovarian cancer [24, 73-76].

The same is true for the FAK pathway. Gene expression related to the FAK pathway was incorporated as a variable in this examination based on reports and previous research indicating that recurrence of ovarian cancer, treatment resistance, and CSCs are related to FAK activity $[24,77-80]$.

Thus, sensitivity and resistance to platinum-containing drugs can be predicted by focusing on metabolic genes and groups of genes related to FAK activity. As a result, the possibility of predicting the prognosis was shown in this examination. Based on this study, metabolism and the FAK pathway may be potential therapeutic targets in the future. In fact, in the test case, the examination using ovarian clear cell carcinoma cell lines, which are likely to be chemotherapy-resistant, showed a synergistic effect of inhibiting glutamine metabolism and the FAK pathway [24]. However, metabolic activity is determined by the overall balance and not only by high or low levels of each group of genes; thus, suggestions for treatment targeting specific gene expression levels have not been determined from this examination. Also, there are limitations from a selection bias and a small sample size.

We believe CSC-like properties are a useful model which gives us insight into chemo-resistance. Especially, we assumed that investigating CSC-like properties of clear cell carcinoma could give us insight into platinum resistance because most of the patients with ovarian clear cell carcinoma are platinum-resistant. We conducted this study to ensure the results we obtained from our previous in-vitro studies. However, there is a possibility that mechanisms of platinum resistance in serous carcinoma is different from that in clear cell carcinoma. In the future, new targets for drug discovery are expected to be found by focusing on metabolism-related genes and FAK activity in treatment-resistant ovarian cancer.

\section{Abbreviations}

FAK: Focal adhesion kinase; FIGO: International Federation of Gynecology and Obstetrics; HGSC: High-grade serous carcinoma; PARP: Poly (ADP-ribose) polymerase; TCGA: The Cancer Genome Atlas; RNA-seq: RNA sequencing; PFI: Platinum free interval; PFS: Progression free survival; OS: Overall survival.

\section{Supplementary Information}

The online version contains supplementary material available at https://doi. org/10.1186/s12885-021-09148-X.

Additional file 1: Figure S1. Throughput output of raw and trimmed data.Analyses were successfully performed on all 16 paired-ends samples.

Additional file 2: Figure S2. Q30 score of raw and trimmed data. Figure shows the Q30 percentage (\% of bases with quality over phred score 30 ) of each sample's raw and trimmed data.

Additional file 3: Figure S3. Overall read mapping ratio. Trimmed reads are mapped to reference genome with HISAT2 [81]. Figure shows the overall read mapping ratio, the ratio of mapped reads to trimmed reads.

Additional file 4: Figure S4. Distribution of the Expression of Each Gene in Each Cluster. (a) The distribution of the expression of each gene in cluster 1. (b) The distribution of the expression of each gene in cluster 2. (c) The mean expression level of each gene in each cluster. Regarding metabolic genes, both high and low expression levels and the overall balance were involved in the metabolic phenotype. Therefore, in this examination, the difference between these 2 groups was unclear

Additional file 5: Table S1. Clinical Specimen Data. 


\section{Acknowledgements}

This study was supported by JSPS KAKENHI Grant Number JP 19K18703.

\section{Authors' contributions}

MS, KH and KF designed the study. MS analyzed the patient data, and a major contributor in writing the manuscript. MS, SS, DS, MH, AO and MM acquired the patient data. AY, AK, HY, KH and KF interpreted the data. MS, KH and KF confirm the authenticity of all the raw data. All authors read and approved the final manuscript.

\section{Funding}

This study was supported by JSPS KAKENHI Grant Number JP 19K18703. The funding body had no role in the design of the study, collection, analysis, and interpretation of data, and in writing the manuscript.

\section{Availability of data and materials}

The datasets used and/or analyzed during the current study are available from the corresponding author on reasonable request.

\section{Declarations}

\section{Ethics approval and consent to participate}

All human-related methods were carried out in accordance with relevant guidelines and regulations. The study was approved by the Institutional Review Board of Saitama Medical University International Medical Center (approval no. 13-165). Tumor specimens were available from the patients who underwent surgery for primary ovarian cancer with their informed consent in the previous study (no. 12-096). Written informed consent from each patient was obtained.

\section{Consent for publication}

Written informed consent for publication from each patient was obtained in the previous study (no. 12-096).

\section{Competing interests}

The authors declare that they have no competing interests.

Received: 11 July 2021 Accepted: 22 December 2021

Published online: 13 January 2022

\section{References}

1. Heintz AP, Odicino F, Maisonneuve P, Quinn MA, Benedet JL, Creasman WT, et al. Carcinoma of the ovary. FIGO 26th Annual Report on the Results of Treatment in Gynecological Cancer. Int J Gynaecol Obstet. 2006;95(Suppl 1):S161-92.

2. Jayson GC, Kohn EC, Kitchener HC, Ledermann JA. Ovarian cancer. Lancet. 2014;384(9951):1376-88.

3. Menon U, Karpinskyj C, Gentry-Maharaj A. Ovarian cancer prevention and screening. Obstet Gynecol. 2018;131(5):909-27.

4. Burger RA, Brady MF, Bookman MA, Fleming GF, Monk BJ, Huang $H$, et al. Incorporation of bevacizumab in the primary treatment of ovarian cancer. N Engl J Med. 2011;365(26):2473-83.

5. Bickell NA, Egorova N, Prasad-Hayes M, Franco R, Howell EA, Wisnivesky $J$, et al. Secondary surgery versus chemotherapy for recurrent ovarian cancer. Am J Clin Oncol. 2018;41(5):458-64.

6. Bristow RE, Tomacruz RS, Armstrong DK, Trimble EL, Montz FJ. Survival effect of maximal cytoreductive surgery for advanced ovarian carcinoma during the platinum era: a meta-analysis. J Clin Oncol. 2002;20(5):1248-59.

7. Lheureux S, Braunstein M, Oza AM. Epithelial ovarian cancer: evolution of management in the era of precision medicine. CA Cancer J Clin. 2019;69(4):280-304.

8. Coleman RL, Spirtos NM, Enserro D, Herzog TJ, Sabbatini P, Armstrong DK, et al. Secondary surgical cytoreduction for recurrent ovarian cancer. N Engl J Med. 2019;381(20):1929-39.

9. Hoppenot C, Eckert MA, Tienda SM, Lengyel E. Who are the longterm survivors of high grade serous ovarian cancer? Gynecol Oncol. 2018;148(1):204-12.
10. Coleman RL, Fleming GF, Brady MF, Swisher EM, Steffensen KD, Friedlander M, et al. Veliparib with first-line chemotherapy and as maintenance therapy in ovarian cancer. N Engl J Med. 2019;381(25):2403-15.

11. González-Martín A, Pothuri B, Vergote I, DePont Christensen R, Graybill W, Mirza MR, et al. Niraparib in patients with newly diagnosed advanced ovarian cancer. N Engl J Med. 2019;381(25):2391-402.

12. Ledermann J, Harter P, Gourley C, Friedlander M, Vergote I, Rustin G, et al. Olaparib maintenance therapy in patients with platinum-sensitive relapsed serous ovarian cancer: a preplanned retrospective analysis of outcomes by BRCA status in a randomised phase 2 trial. Lancet Oncol. 2014;15(8):852-61.

13. Mirza MR, Monk BJ, Herrstedt J, Oza AM, Mahner S, Redondo A, et al. Niraparib maintenance therapy in platinum-sensitive, recurrent ovarian cancer. N Engl J Med. 2016;375(22):2154-64.

14. Moore K, Colombo N, Scambia G, Kim BG, Oaknin A, Friedlander M, et al. Maintenance olaparib in patients with newly diagnosed advanced ovarian cancer. N Engl J Med. 2018;379(26):2495-505.

15. Moore KN, Secord AA, Geller MA, Miller DS, Cloven N, Fleming GF, et al. Niraparib monotherapy for late-line treatment of ovarian cancer (QUADRA): a multicentre, open-label, single-arm, phase 2 trial. Lancet Oncol. 2019;20(5):636-48.

16. Pujade-Lauraine E, Ledermann JA, Selle F, Gebski V, Penson RT, Oza AM, et al. Olaparib tablets as maintenance therapy in patients with platinumsensitive, relapsed ovarian cancer and a BRCA1/2 mutation (SOLO2/ ENGOT-Ov21): a double-blind, randomised, placebo-controlled, phase 3 trial. Lancet Oncol. 2017;18(9):1274-84.

17. Ray-Coquard I, Pautier P, Pignata S, Pérol D, González-Martín A, Berger $\mathrm{R}$, et al. Olaparib plus bevacizumab as first-line maintenance in ovarian cancer. N Engl J Med. 2019;381(25):2416-28.

18. Salwa A, Ferraresi A, Chinthakindi M, Vallino L, Vidoni C, Dhanasekaran DN, et al. BECN1 and BRCA1 deficiency sensitizes ovarian cancer to platinum therapy and confers better prognosis. Biomedicines. 2021;9(2):207.

19. Zhang G, Zhang J, Zhu Y, Liu H, Shi Y, Mi K, et al. Association of somatic mutations in BRCA2 BRC domain with chemotherapy sensitivity and survival in high grade serous ovarian cancer. Exp Cell Res. 2021;406(1):112742.

20. Barbato L, Bocchetti M, Di Biase A, Regad T. Cancer stem cells and targeting strategies. Cells. 2019;8(8):926.

21. Batlle E, Clevers H. Cancer stem cells revisited. Nat Med. 2017;23(10):1124-34.

22. Li L, Bhatia R. Stem cell quiescence. Clin Cancer Res. 2011;17(15):4936-41.

23. Muñoz-Galván S, Carnero A. Targeting cancer stem cells to overcome therapy resistance in ovarian cancer. Cells. 2020;9(6):1402.

24. Sato M, Kawana K, Adachi K, Fujimoto A, Yoshida M, Nakamura H, et al. Targeting glutamine metabolism and the focal adhesion kinase additively inhibits the mammalian target of the rapamycin pathway in spheroid cancer stem-like properties of ovarian clear cell carcinoma in vitro. Int J Oncol. 2017;50(4):1431-8.

25. Cristiano S, Leal A, Phallen J, Fiksel J, Adleff V, Bruhm DC, et al. Genomewide cell-free DNA fragmentation in patients with cancer. Nature. 2019:570(7761):385-9.

26. Hossain MA, Saiful Islam SM, Quinn JMW, Huq F, Moni MA. Machine learning and bioinformatics models to identify gene expression patterns of ovarian cancer associated with disease progression and mortality. J Biomed Inform. 2019;100:103313.

27. Huang C, Clayton EA, Matyunina LV, McDonald LD, Benigno BB, Vannberg $F$, et al. Machine learning predicts individual cancer patient responses to therapeutic drugs with high accuracy. Sci Rep. 2018;8(1):16444.

28. Huang C, Mezencev R, McDonald JF, Vannberg F. Open source machinelearning algorithms for the prediction of optimal cancer drug therapies. PLoS One. 2017;12(10):e0186906.

29. Kawakami E, Tabata J, Yanaihara N, Ishikawa T, Koseki K, lida Y, et al. Application of artificial intelligence for preoperative diagnostic and prognostic prediction in epithelial ovarian cancer based on blood biomarkers. Clin Cancer Res. 2019;25(10):3006-15.

30. Lu TP, Kuo KT, Chen CH, Chang MC, Lin HP, Hu YH, et al. Developing a prognostic gene panel of epithelial ovarian cancer patients by a machine learning model. Cancers (Basel). 2019;11(2):270.

31. Mucaki EJ, Zhao JZL, Lizotte DJ, Rogan PK. Predicting responses to platin chemotherapy agents with biochemically-inspired machine learning. Signal Transduct Target Ther. 2019;4:1. 
32. Paik ES, Lee JW, Park JY, Kim JH, Kim M, Kim TJ, et al. Prediction of survival outcomes in patients with epithelial ovarian cancer using machine learning methods. J Gynecol Oncol. 2019;30(4):e65.

33. Shinagare AB, Balthazar $P, I p ~ I K$, Lacson R, Liu J, Ramaiya N, et al. High-grade serous ovarian cancer: use of machine learning to predict abdominopelvic recurrence on $\mathrm{CT}$ on the basis of serial cancer antigen 125 levels. J Am Coll Radiol. 2018;15(8):1133-8.

34. Song HJ, Yang ES, Kim JD, Park CY, Kyung MS, Kim YS. Best serum biomarker combination for ovarian cancer classification. Biomed Eng Online. 2018;17(Suppl 2):152.

35. Tseng CJ, Lu CJ, Chang CC, Chen GD, Cheewakriangkrai C. Integration of data mining classification techniques and ensemble learning to identify risk factors and diagnose ovarian cancer recurrence. Artif Intell Med. 2017;78:47-54.

36. Wang $X$, Han L, Zhou L, Wang L, Zhang LM. Prediction of candidate RNA signatures for recurrent ovarian cancer prognosis by the construction of an integrated competing endogenous RNA network. Oncol Rep. 2018;40(5):2659-73.

37. Ikeda Y, Sato S, Yabuno A, Shintani D, Ogasawara A, Miwa M, et al. High expression of maternal embryonic leucine-zipper kinase (MELK) impacts clinical outcomes in patients with ovarian cancer and its inhibition suppresses ovarian cancer cells growth ex vivo. J Gynecol Oncol. 2020;31(6):e93.

38. Schwartz LH, Litière S, de Vries E, Ford R, Gwyther S, Mandrekar S, et al. RECIST 1.1-Update and clarification: from the RECIST committee. Eur J Cancer. 2016;62:132-7.

39. Dockery LE, Rubenstein AR, Ding K, Mashburn SG, Burkett WC, Davis AM, et al. Extending the platinum-free interval: the impact of omitting 2 nd line platinum chemotherapy in intermediate platinum-sensitive ovarian cancer. Gynecol Oncol. 2019;155(2):201-6.

40. Milanowski $Ł$, Hoffman-Zacharska D, Geremek M, Friedman A, Figura M, Koziorowski D. The matter of significance - Has the p.(Glu121Lys) variant of TOR1A gene a pathogenic role in dystonia or Parkinson disease? J Clin Neurosci. 2020;72:501-3.

41. Akbani R, Ng PK, Werner HM, Shahmoradgoli M, Zhang F, Ju Z, et al. A pan-cancer proteomic perspective on The Cancer Genome Atlas. Nat Commun. 2014;5:3887.

42. Bao M, Zhang L, Hu Y. Novel gene signatures for prognosis prediction in ovarian cancer. J Cell Mol Med. 2020;24(17):9972-84.

43. Dellinger $A E$, Nixon $A B$, Pang $H$. Integrative pathway analysis using graphbased learning with applications to TCGA colon and ovarian data. Cancer Inform. 2014;13(Suppl 4):1-9.

44. He Z, Zhang J, Yuan X, Liu Z, Liu B, Tuo S, et al. Network based stratification of major cancers by integrating somatic mutation and gene expression data. PLoS One. 2017;12(5):e0177662.

45. Hillman RT, Chisholm GB, Lu KH, Futreal PA. Genomic rearrangement signatures and clinical outcomes in high-grade serous ovarian cancer. J Natl Cancer Inst. 2018;110(3):265-72.

46. Lin H, Wang J, Wen X, Wen Q, Huang S, Mai Z, et al. A prognosis-predictive nomogram of ovarian cancer with two immune-related genes: CDC20B and PNPLA5. Oncol Lett. 2020;20(5):204.

47. Niu Y, Sun W, Chen K, Fu Z, Chen Y, Zhu J, et al. A novel scoring system for pivotal autophagy-related genes predicts outcomes after chemotherapy in advanced ovarian cancer patients. Cancer Epidemiol Biomarkers Prev. 2019:28(12):2106-14.

48. Qin L, LiT, Liu Y. High SLC4A11 expression is an independent predictor for poor overall survival in grade $3 / 4$ serous ovarian cancer. PLoS One. 2017;12(11):e0187385.

49. Sun T, Yang Q. Chemoresistance-associated alternative splicing signatures in serous ovarian cancer. Oncol Lett. 2020;20(1):420-30.

50. Wang R, Ye XH, Zhao XL, Liu JL, Zhang CY. Development of a five-gene signature as a novel prognostic marker in ovarian cancer. Neoplasma. 2019;66(3):343-9.

51. Yan S, Fang J, Chen Y, Xie Y, Zhang S, Zhu X, et al. Comprehensive analysis of prognostic gene signatures based on immune infiltration of ovarian cancer. BMC Cancer. 2020;20(1):1205.

52. Saji H, Tsuboi M, Shimada Y, Kato Y, Hamanaka W, Kudo Y, et al. Gene expression profiling and molecular pathway analysis for the identification of early-stage lung adenocarcinoma patients at risk for early recurrence. Oncol Rep. 2013;29(5):1902-6.

53. Babyak MA. What you see may not be what you get: a brief, nontechnical introduction to overfitting in regression-type models. Psychosom Med. 2004;66(3):411-21.
54. Altman BJ, Stine ZE, Dang CV. From Krebs to clinic: glutamine metabolism to cancer therapy. Nat Rev Cancer. 2016;16(10):619-34.

55. Desgrosellier JS, Cheresh DA. Integrins in cancer: biological implications and therapeutic opportunities. Nat Rev Cancer. 2010;10(1):9-22.

56. Pavlova NN, Thompson CB. The emerging hallmarks of cancer metabolism. Cell Metab. 2016;23(1):27-47.

57. Sancho P, Barneda D, Heeschen C. Hallmarks of cancer stem cell metabolism. Br J Cancer. 2016;114(12):1305-12.

58. Yang M, Vousden $\mathrm{KH}$. Serine and one-carbon metabolism in cancer. Nat Rev Cancer. 2016;16(10):650-62

59. Tucker SL, Gharpure K, Herbrich SM, Unruh AK, Nick AM, Crane EK, et al. Molecular biomarkers of residual disease after surgical debulking of highgrade serous ovarian cancer. Clin Cancer Res. 2014;20(12):3280-8.

60. Tothill RW, Tinker AV, George J, Brown R, Fox SB, Lade S, et al. Novel molecular subtypes of serous and endometrioid ovarian cancer linked to clinical outcome. Clin Cancer Res. 2008;14(16):5198-208.

61. Tischler J, Gruhn WH, Reid J, Allgeyer E, Buettner F, Marr C, et al. Metabolic regulation of pluripotency and germ cell fate through a-ketoglutarate. EMBO J. 2019;38(1):e99518.

62. Knijnenburg TA, Wang L, Zimmermann MT, Chambwe N, Gao GF, Cherniack AD, et al. Genomic and molecular landscape of DNA damage repair deficiency across the cancer genome atlas. Cell Rep. 2018;23(1):239-54.e6.

63. Lu M, Fan Z, Xu B, Chen L, Zheng X, Li J, et al. Using machine learning to predict ovarian cancer. Int J Med Inform. 2020;141:104195.

64. Lu J, Li HM, Cai SQ, Zhao SH, Ma FH, Li YA, et al. Prediction of platinum-based chemotherapy response in advanced high-grade serous ovarian cancer: ADC histogram analysis of primary tumors. Acad Radiol. 2021;28(3):e77-85.

65. Mairinger F, Bankfalvi A, Schmid KW, Mairinger E, Mach P, Walter RF, et al. Digital immune-related gene expression signatures in high-grade serous ovarian carcinoma: developing prediction models for platinum response. Cancer Manag Res. 2019;11:9571-83.

66. Murakami R, Matsumura N, Brown JB, Wang Z, Yamaguchi K, Abiko K, et al. Prediction of taxane and platinum sensitivity in ovarian cancer based on gene expression profiles. Gynecol Oncol. 2016;141(1):49-56.

67. Telli ML, Timms KM, Reid J, Hennessy B, Mills GB, Jensen KC, et al. Homologous Recombination Deficiency (HRD) score predicts response to platinum-containing neoadjuvant chemotherapy in patients with triple-negative breast cancer. Clin Cancer Res. 2016;22(15):3764-73.

68. Tumiati M, Hietanen S, Hynninen J, Pietila E, Farkkila A, Kaipio K, et al. A functional homologous recombination assay predicts primary chemotherapy response and long-term survival in ovarian cancer patients. Clin Cancer Res. 2018;24(18):4482-93.

69. van Zyl B, Tang D, Bowden NA. Biomarkers of platinum resistance in ovarian cancer: what can we use to improve treatment. Endocr Relat Cancer. 2018;25(5):R303-R18.

70. Wu C, He L, Wei Q, Li Q, Jiang L, Zhao L, et al. Bioinformatic profiling identifies a platinum-resistant-related risk signature for ovarian cancer. Cancer Med. 2020;9(3):1242-53.

71. Yamawaki K, Mori Y, Sakai H, Kanda Y, Shiokawa D, Ueda H, et al. Integrative analyses of gene expression and chemosensitivity of patient-derived ovarian cancer spheroids link G6PD-driven redox metabolism to cisplatin chemoresistance. Cancer Lett. 2021;521:29-38.

72. Shannon NB, Tan LLY, Tan QX, Tan JW, Hendrikson J, Ng WH, et al. A machine learning approach to identify predictive molecular markers for cisplatin chemosensitivity following surgical resection in ovarian cancer. Sci Rep. 2021;11(1):16829.

73. Durán RV, Oppliger W, Robitaille AM, Heiserich L, Skendaj R, Gottlieb E, et al. Glutaminolysis activates Rag-mTORC1 signaling. Mol Cell. 2012;47(3):349-58.

74. Jin L, Alesi GN, Kang S. Glutaminolysis as a target for cancer therapy. Oncogene. 2016;35(28):3619-25.

75. Stincone A, Prigione A, Cramer T, Wamelink MM, Campbell K, Cheung E, et al. The return of metabolism: biochemistry and physiology of the pentose phosphate pathway. Biol Rev Camb Philos Soc. 2015;90(3):927-63.

76. Xie H, Hanai J, Ren JG, Kats L, Burgess K, Bhargava P, et al. Targeting lactate dehydrogenase--a inhibits tumorigenesis and tumor progression in mouse models of lung cancer and impacts tumor-initiating cells. Cell Metab. 2014;19(5):795-809.

77. Chen CH, Shyu MK, Wang SW, Chou CH, Huang MJ, Lin TC, et al. MUC20 promotes aggressive phenotypes of epithelial ovarian cancer cells via activation of the integrin $\beta 1$ pathway. Gynecol Oncol. 2016;140(1):131-7. 
78. McGrail DJ, Khambhati NN, Qi MX, Patel KS, Ravikumar N, Brandenburg $\mathrm{CP}$, et al. Alterations in ovarian cancer cell adhesion drive taxol resistance by increasing microtubule dynamics in a FAK-dependent manner. Sci Rep. 2015;5:9529.

79. Shah NR, Tancioni I, Ward KK, Lawson C, Chen XL, Jean C, et al. Analyses of merlin/NF2 connection to FAK inhibitor responsiveness in serous ovarian cancer. Gynecol Oncol. 2014;134(1):104-11.

80. Tancioni I, Uryu S, Sulzmaier FJ, Shah NR, Lawson C, Miller NL, et al. FAK Inhibition disrupts a $\beta 5$ integrin signaling axis controlling anchorage-independent ovarian carcinoma growth. Mol Cancer Ther. 2014;13(8):2050-61.

81. Kim D, Langmead B, Salzberg SL. HISAT: a fast spliced aligner with low memory requirements. Nat Methods. 2015;12(4):357-60.

\section{Publisher's Note}

Springer Nature remains neutral with regard to jurisdictional claims in published maps and institutional affiliations.

- fast, convenient online submission

- thorough peer review by experienced researchers in your field

- rapid publication on acceptance

- support for research data, including large and complex data types

- gold Open Access which fosters wider collaboration and increased citations

- maximum visibility for your research: over $100 \mathrm{M}$ website views per year

At BMC, research is always in progress.

Learn more biomedcentral.com/submissions 